\title{
OCCURRENCE AND DEGREE OF LIIOPSOAS MUSCLE CONTRACTURE IN REEULAR MALE SQUASH PLAYERS
}

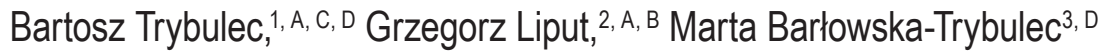 \\ 1 Jagiellonian University Medical College, Faculty of Health Sciences, Department of Physiotherapy, Institute of Physiotherapy, Kraków, Poland \\ ${ }^{2}$ Graduate of Physiotherapy, Jagiellonian University Medical College, Faculty of Health Sciences, Kraków, Poland \\ ${ }^{3}$ Jagiellonian University Medical College, Faculty of Health Sciences, Department of Medical Physiology, Institute of Physiotherapy, \\ Kraków, Poland

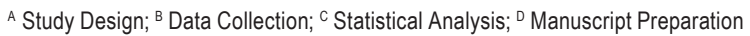

\author{
Address for correspondence: \\ Bartosz Trybulec \\ Jagiellonian University Medical College, Institute of Physiotherapy \\ Medyczna 9, 30-688 Kraków, Poland \\ E-mail: bartosz.trybulec@uj.edu.pl
}

Ahstract Introduction: Sprints combined with changes in direction and repeated lunges are the most frequent movements during a squash game. These motions overload the iliopsoas muscle which may cause a lot of microinjuries. Accumulating microinjuries combined with a lack of stretching exercises may lead to iliopsoas contracture.

Aim of the study: Assessment of the frequency and degree of iliopsoas contracture in regular squash players.

Material and methods: The experimental group comprised 25 regular squash players (minimum 2 years of playing at least twice a week) and 21 non-players (control group). A modified Thomas Test was used to assess iliopsoas contracture using goniometric and linear measurements.

Results: lliopsoas contracture was observed in $96 \%$ of the squash players and $66.7 \%$ of the non-players $(p=0.0089$ ). The degree of muscle contracture in the goniometric measurement was greater in squash players than in the non-players in both the left $(p=0.0303)$ and right $(p=0.0007)$ iliopsoas muscles. There were no statistically significant differences in the linear measurement.

Conclusions: There is a positive relationship between regularly playing squash and the frequency of iliopsoas contracture occurrence being significantly greater in squash players than in non-players.

Key WOrds squash, iliopsoas, contracture, modified Thomas test

\section{Introduction}

Squash is a racquet sport played by two players on a court surrounded by walls on four sides, using a hollow rubber ball. The ball is hit by the players alternately using a racquet. The ball can bounce once on the floor and any number of times from the court walls. The main strategy of a squash player is to force the opponent to move around 
the entire area to be played. If this strategy is successfully implemented, the opponent travels a greater distance and subjects the body to a greater load (Vuckovic, Dezman, Erculj, Kovacic, Pers, 2011).

According to C. Eubank and N. Messenger (2000), squash players perform an average of 2,866 steps per match (580 steps per set). $74.4 \%$ of them contain a flight phase. This indicates that the game is very dynamic. Performing such intensive work can result in increased muscle stiffness and can lead to contracture. However, this is not a physiological adaptation, because adequate flexibility is an innate property of the tissues of the myofascial system, determining the normal range of motion and thus the functioning of the joints and the entire limb (Thacker, Gilchrist, Stroup, Kimsey, 2004).

Commonly performed movements in squash are the lunge and sprint. Repeated executions burden the iliopsoas muscle. Repetitive eccentric work changes the movement pattern and increases muscle stiffness. According to E.P. Roetert, T.S. Ellenbecker and S.W. Brown (2020), these are early adaptations that appear in regular players before the onset of clinical symptoms.

Imbalance between the iliopsoas and glutei muscles can contribute to an increase in the load on the front of the hip. In this way, the anterior glide of the femoral head increases, due to reduced exploitation of the gluteal muscles during hip extension and the iliopsoas muscle during flexion. It is likely that the increase in forces acting on the front of the hip is caused by an increase in the frontal slip. This subtle instability, together with placing the hip in an upright position, can cause serious injuries such as detachment of the labrum. Based on the above information it is assumed that an appropriate distribution of forces on the hip joint can ensure coordination and elasticity of the iliopsoas and gluteus maximus, thanks to which the right strength for the hip movements and the correct position of the joint can be obtained (Lewis, Sahrmann, Moran, 2007; Shindle, Ranawat, Kelly, 2006; Carlos, Guanche, Robby, Sikka, 2005).

A squash player is subjected to a variety loads during a match. Game strategy demands specific body positions to make it easier to cope with this task. The hip, knee and ankle joints during a lunge (fundamental position in squash) are in a flexion position (Gyoung-Mo, Sung-Min, 2015) (Figure 1). The aim of this study was to assess the incidence and degree of iliopsoas muscle contracture in regular squash players.

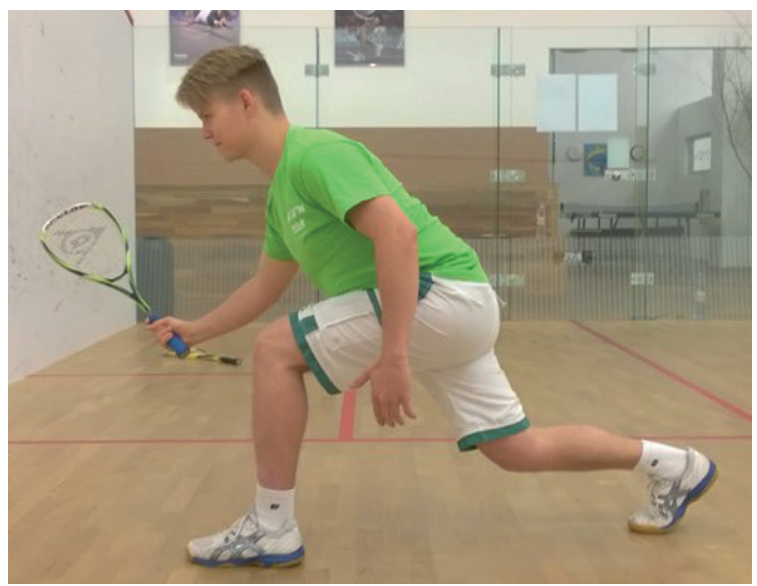

Figure 1. Lunge - main position for receiving the ball in the squash 


\section{Material and methods}

The study consisted of 54 men, recruited personally by the authors and through social media, comprising 32 squash players and 22 non-players. At the outset, an original questionnaire was completed by each participant that contained questions about age, weight and height, the frequency of squash trainings, and the possible presence of specific exclusion criteria. The criteria for joining the study group was at least 2 years of practicing squash and a frequency of at least 2 trainings a week. The exclusion criteria were:

1. For the study group:

- squash playing period $<2$ years,

- number of trainings $\leq 2$ per week,

- iliopsoas muscle/hip region injuries in the past,

- age $<18$ or $>40$ years.

2. For the control group:

- practicing squash,

- doing sports requiring intensive usage of the iliopsoas muscle,

- iliopsoas muscle/hip region injury in the past,

- age $<18$ or $>40$ years old.

Ultimately, the study group consisted of 25 squash players, and 21 non-players in the control group. In the study group the average age was 28.8 years, average body height was $179.8 \mathrm{~cm}$ and average body weight was $77.9 \mathrm{~kg}$. In the control group the average age was 25.1 years, average body height was $177.2 \mathrm{~cm}$ and average body weight was $76.5 \mathrm{~kg}$ (Table 1).

Table 1. Descriptive statistics of somatic parameters in both study groups

\begin{tabular}{|c|c|c|c|c|c|c|c|c|c|}
\hline \multirow{2}{*}{ Parameter } & \multicolumn{4}{|c|}{ Squash group $(n=25)$} & \multirow{2}{*}{$\mathrm{p}$} & \multicolumn{4}{|c|}{ Control group $(n=21)$} \\
\hline & mean & SD & Min & Max & & mean & SD & Min & Max \\
\hline Body weight (kg) & 77.80 & 7.56 & 66 & 92 & 0.5723 & 76.5 & 9.20 & 59 & 100 \\
\hline Body height $(\mathrm{cm})$ & 179.80 & 4.85 & 168 & 190 & 0.0674 & 177.2 & 4.48 & 170 & 185 \\
\hline Age (yrs) & 28.84 & 6.32 & 19 & 40 & 0.0796 & 25.1 & 4.90 & 20 & 36 \\
\hline
\end{tabular}

The functional measurement comprised a modified Thomas test (Figures 2 and 3 ) in a supine position on a table, and in the case of a positive result, a goniometric and linear measurement of the degree of iliopsoas muscle contracture (Wakefield, Halls, Difilippo, Cottrell, 2015; Whiting, Zernicke, 2008). The test consisted of lifting the knee to the chest until the lumbar lordosis was compensated. The test result was considered positive when the thigh of resting leg was lifted above the table surface. In this case, the angular measurement between the thigh axis and the table plane was taken assuming the greater trochanter as the axis of the rotation (Figure 2) and the measurement of the distance between the thigh and the table plane at the level of the lateral epicondyle of the femur (Figure 3). 


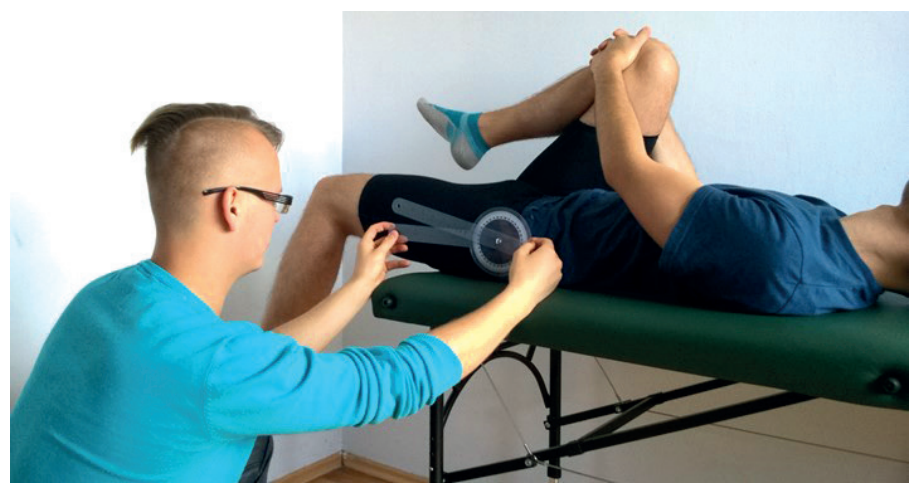

Figure 2. Modified Thomas test: goniometric measurement

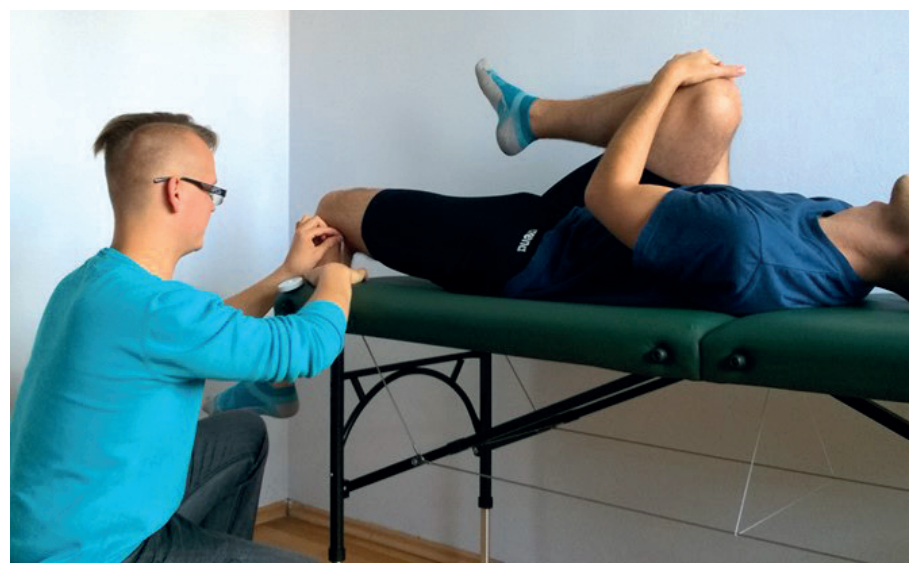

Figure 3. Modified Thomas test: tape measurement

Statistical data analysis of the obtained results was carried out using STATISTICA v.12.0 software (StatSoft Inc.). Compatibility of variable distribution with a normal distribution was tested using the Shapiro-Wilk test. To compare the means of the independent variables, Student's t-test and Mann-Whitney $U$ tests were used, and to assess differences in the occurrence of contracture in both study groups a Chi Square test was used. The results were considered significant at $p<0.05$.

\section{Results}

In the study group, iliopsoas muscle contracture was observed in 24 of the 25 squash players (in 20 of them bilaterally). In the control group contracture was noticed in 14 of the 21 men (bilaterally in 10 of them). After analysis using the Chi Square test, a statistically significant relationship was stated between playing squash and the occurrence of iliopsoas muscle contracture $(p=0.0089)$ (Table 2$)$. 
Table 2. Distribution of the frequency of contracture in the study and control groups

\begin{tabular}{lcrc}
\hline \multicolumn{1}{c}{ Groups } & Contracture & N & Percent \\
\hline \multirow{2}{*}{ Study group } & no & 1 & 4.0 \\
& yes & 24 & 96.0 \\
\hline \multirow{2}{*}{ Control group } & no & 7 & 33.3 \\
& yes & 14 & 66.7 \\
\hline Chi Square test $=6.84 ; p=0.0089$ & &
\end{tabular}

Table 3 presents a comparison of the degrees of iliopsoas muscle contracture in both groups. Analysis by Student's t-test (Left [deg] and Right [cm] measurement) and Mann-Whitney $U$ test (Right [deg] and Left [cm] measurement) showed a statistical significance for differences between angular measurements $(p=0.0303$ for the left lower limb, 0.0007 for the right lower limb).

Tahle 3. Comparison of the degree of iliopsoas muscle contracture for lower limbs in both study groups in the goniometric (deg) and linear $(\mathrm{cm})$ tests

\begin{tabular}{|c|c|c|c|c|c|c|c|c|c|}
\hline \multirow{2}{*}{ Limb } & \multicolumn{4}{|c|}{ Study group $(n=24)$} & \multirow{2}{*}{ p } & \multicolumn{4}{|c|}{ Control group $(n=14)$} \\
\hline & $\bar{x}$ & SD & $\mathrm{Me}$ & Max & & $\bar{x}$ & $\mathrm{SD}$ & $\mathrm{Me}$ & Max \\
\hline Left (deg) & 11.45 & 6.21 & 13.00 & 20 & $0.0303(\mathrm{t})$ & 7.07 & 4.94 & 7.50 & 15 \\
\hline Left $(\mathrm{cm})$ & 3.52 & 2.98 & 4.50 & 9 & $0.9881(U)$ & 3.68 & 3.47 & 3.25 & 12 \\
\hline Right (deg) & 11.08 & 5.61 & 10.00 & 20 & $0.0007(U)$ & 5.29 & 3.87 & 5.50 & 14 \\
\hline Right (cm) & 4.18 & 3.25 & 4.75 & 12 & $0.1933(t)$ & 2.86 & 2.44 & 2.50 & 7 \\
\hline
\end{tabular}

$\bar{x}$ - mean, SD - standard deviation, Me - median, $\mathrm{t}$ - t-Student test, $\mathrm{U}$ - Mann-Whitney test, $\mathrm{p}$ - level of significance.

\section{Discussion}

This research is pioneering as no publication available to date have addressed the topic of iliopsoas muscle contracture in squash players. While publications related to other racquet sports exist, such as in tennis and badminton, they can be considered an important element of this discussion due to the lack of published research on squash (Young et al., 2014; Kovacs, 2006; Girard et al., 2007; Ellenbecker et al., 2007).

The incidence of iliopsoas muscle contracture was investigated in both study groups and a statistically significant $(p=0.0089)$ difference found between the $96.3 \%$ of the squash players and $66.7 \%$ of the nonplayers with this condition. These results differed from those obtained by S.W. Young et al. (2014) who examined 125 professional tennis players using the Thomas test and found contracture of the iliopsoas muscle in $51 \%$ of the players. In addition, the positive result of the hip flexor test was significantly correlated with abdominal muscle overload in female players (Young et al., 2014). Results of the study conducted by W.B. Kibler and T.J. Chandler (2003) showed that the myofascial system in women is more flexible than in men. Thus, iliopsoas muscle contracture may be more common in male squash players than in tennis players. The techniques of squash require the lunge, while in tennis this is almost absent. The eccentric work generated during the lunge is a significant burden on the lower limb, especially for the iliopsoas muscle. Thus, contracture may occur more often in squash players. Another difference between these sports is the different dynamics of the game of squash (Kovacs, 2006; Girard et al., 2007). 
The occurrence of iliopsoas muscle contracture in squash players as well as in other sports (tennis, handball, volleyball, golf, swimming, gymnastics, dancing, judo, weightlifting, and even motocross) may be related to the theory of microtrauma summation. This mechanism may lead to the appearance of musculoskeletal disorders: trauma from overload (e.g. fatigue fracture) or chronic soft tissue rheumatism (e.g. tennis elbow, iliac-lumbar muscle tendonitis). Micro-injuries arising in muscles as a result of repetitive eccentric work can lead to muscle contracture and consequently, to an increase in the incidence of serious injuries (Roetert et al., 2020; Ho, Lee, Chang, Chen, Huang, 2020; Kajetanek et al., 2016; Russel, Wiese-Bjornstal, 2015; Doyscher, Kraus, Finke, Scheibel, 2014; Váczi, Rácz, Hortobágyi, Tihanyi, 2013).

On the basis of the results of this study, a significant reduction in iliopsoas muscle function was observed in the form of contracture occurring in regular squash players. It can therefore be assumed that functional changes should also be observed at the structural level. This conclusion is not confirmed by the results obtained by G. Nketiah et al. (2015) who assessed the condition of the iliopsoas muscle in squash players using magnetic resonance imaging. The results showed that there are no significant structural differences between squash players and the control group. This is probably due to the lack of exact criteria for inclusion in the study group (e.g. a minimum of 2 years of squash, at least 2 times a week) or the small sample of 10 patients. Confirmation of the presence of structural changes requires further research.

In this study, goniometric and linear measurements were used to assess the degree of iliopsoas muscle contracture in both sides. This course of research was intended to check for possible convergence of these types of measurements. Statistical analysis showed a significant difference between the degree of contracture in the goniometric measurement in both the left $(p=0.0303)$ and right lower limbs between the test group and the control group $(p=0.0007)$. In turn, statistical analysis of linear measurements did not show significant differences in contracture between the groups. This discrepancy in the results may be caused by the methodology of the goniometric and linear measurements. The goniometer has a larger span scale, so the relative approximation error during measurement may be smaller with a higher measurement compliance factor. Another factor that can affect the measurement results is how the test was designed. Goniometric measurement was performed first. It is therefore possible that the iliopsoas muscle during the goniometric measurement was stretched, which could increase the range of hip joint motion affecting the result of the linear measurement (Young et al., 2014).

Our study has certain limitations that may significantly affect the results. The modified Thomas test has many variables that can distort the result or completely prevent a performance, such as: motion of the lumbar spine, forward or backward tilt of the pelvis, the ability to bend the hip and knee joint, waist circumference, thigh circumference. The factor that most often disturbs the test result is the mobility of the lumbar-pelvic complex. Pelvic inclination can make up for the lack of hip extension and thus compensate for flexion contracture. Thus, the modified Thomas test (MTT) used to investigate iliopsoas muscle contracture may not give reliable results (Vigotsky et al., 2016). Despite the fact that MTT is widely used in orthopedic and physiotherapeutic practice, it shows little credibility if the movement of the lumbar-pelvic complex is not controlled. Proper pelvic stabilization is therefore a key factor for MTT reliability, and this largely depends on the experience of the examiner. The universality of using MTT in clinical practice has been justified in previous studies showing a high consistency between the researchers, between $95 \%$ and $97.6 \%$. The above information allows us to assume that a correctly performed MTT is a reliable for assessing iliopsoas muscle contracture (Ferber, Kendall, McElroy, 2010). 
Another limitation is the relatively small size of both study groups. For a larger number of subjects, it would be possible to use more accurate parametric tests in place of the non-parametric Mann-Whitney $U$ test. There is also the chance that a larger number of groups would result in a greater similarity and thus a greater reliability in the results (Jones, Carley, Harrison, 2003).

The study showed a significantly higher incidence and degree of iliopsoas muscle contracture in squash players compared to the non-players. Therefore, it is worth paying attention to the prevention of this unfavorable compensation in the context of reducing the risk of sport injuries (Jones, Carley, Harrison, 2003). Creating appropriate prevention programs may contribute to minimizing this risk and protect squash players from injuries (Ellenbecker et al., 2007).

\section{Conclusions}

1. Contraction of the iliopsoas muscle occurs significantly more frequently in men regularly practicing squash than in non-players.

2. The degree of iliopsoas muscle contracture in men regularly practicing squash is significantly higher than in the non-players, indicating a lack of targeted stretching exercises to alleviate this condition.

3. Further research is needed on the specific impact of squash on the musculoskeletal system, taking into account more factors and using specialized measuring equipment.

\section{References}

Carlos, A., Guanche, M.D., Robby, S., Sikka, B.A. (2005). Acetabular labral tears with underlying chondromalacia: a possible association with high-level running. Arthroscopy: The Journal of Arthroscopic \& Related Surgery, 21 (5), 580-585. DOI: 10.1016/j.arthro.2005.02.016.

Doyscher, R., Kraus, K., Finke, B., Scheibel, M. (2014). Acute and overuse injuries of the shoulder in sports. Orthopade, 43 (3), $202-208$. DOI: 10.1007/s00132-013-2141-x.

Ellenbecker, T.S., Ellenbecker, G.A., Roetert, E.P., Silva, R.T., Keuter, G., Sperling, F. (2007). Descriptive profile of hip rotation range of motion in elite tennis players and professional baseball pitchers. The American Journal of Sports Medicine, 35 (8), 1371-1376. DOI: $10.1177 / 0363546507300260$.

Eubank, C., Messenger, N. (2000). Dynamic moves and stepping patterns typical to the game of squash. Journal of Sport Sciences, 18, 471-472.

Ferber, R., Kendall, K.D., McElroy, L. (2010). Normative and Critical Criteria for Iliotibial Band and Iliopsoas Muscle Flexibility. Journal of Athletic Training, 45 (4), 344-348.

Girard, O., Chevalier, R., Habrard, M., Sciberras, P., Hot, P., Millet, G.P. (2007). Game analysis and energy requirements of elite squash. The Journal of Strength \& Conditioning Research, 21 (3), 909-914. DOI: 10.1519/R-20306.1.

Gyoung-Mo, K., Sung-Min, H. (2015). Reliability of the modified Thomas test using a lumbo-pelvic stabilization. Journal of Physical Therapy Science, 27 (2), 447-449. DOI: 10.1589/jpts.27.447.

Ho, C.S., Lee, M.C., Chang, C.Y., Chen, W.C., Huang, W.C. (2020). Beneficial effects of a negative ion patch on eccentric exerciseinduced muscle damage, inflammation, and exercise performance in badminton athletes. Chinese Journal of Physiology, 63 (1), 35-42. DOI: 10.4103/CJP.CJP_33_19.

Jones, S., Carley, S., Harrison, M. (2003). An introduction to power and sample size estimation. Emergency Medicine Journal, 20 (5), 453-458. DOI: 10.1136/emj.20.5.453.

Kajetanek, C., Thaunat, M., Guimaraes, T., Carnesecchi, O., Daggett, M., Sonnery-Cottet, B. (2016). Arthroscopic treatment of painful Sinding-Larsen-Johansson syndrome in a professional handball player. Orthopaedics \& Traumatology, 102 (5), 677-680. DOI: 10.1016/j.otsr.2016.05.011.

Kibler, W.B., Chandler, T.J. (2003). Range of motion in junior tennis players participating in an injury risk modification program. Journal of Science and Medicine in Sport, 6 (1), 51-62. DOI: 10.1016/S1440-2440(03)80008-7. 
Kovacs, M.S. (2006). Applied physiology of tennis performance. British Journal of Sports Medicine, 40 (5), 381-386. DOI: 10.1136/ bjsm.2005.023309.

Lewis, C.L., Sahrmann, S.A., Moran, D.W. (2007). Anterior hip joint force increases with hip extension, decreased gluteal force, or decreased iliopsoas force. Journal of Biomechanics, 40 (16), 3725-3731. DOI: 10.1016/j.jbiomech.2007.06.024.

Nketiah, G., Savio, S., Dastidar, P., Nikander, R., Eskola, H., Sievänen, H. (2015). Detection of exercise load-associated differences in hip muscles. Scandinavian Journal of Medicine and Science in Sports, 25 (3), 428-434. DOI: 10.1111/sms.12247.

Roetert, E.P., Ellenbecker, T.S., Brown, S.W. (2000). Shoulder internal and external rotation range of motion in nationally ranked junior tennis players: longitudinal analysis. Journal of Strength \& Conditioning Research, 14, 140-143.

Russell, H.C., Wiese-Bjornstal, D.M. (2015). Narratives of Psychosocial Response to Microtrauma Injury. Sports, 3 (3), 159-177. DOI: 10.3390/sports3030159.

Shindle, M.K., Ranawat, A.S., Kelly, B.T. (2006) Diagnosis and management of traumatic and atraumatic hip instability in the athletic patient. Clinical Journal of Sport Medicine, 25 (2), 309-326. DOI: 10.1016/j.csm.2005.12.003.

Thacker, S.B., Gilchrist, J., Stroup, D.F., Kimsey, C.D. (2004). The impact of stretching on sports injury risk a systematic review of the literature. Medicine \& Science in Sports \& Exercise, 34, 371-378. DOI: 10.1249/01.MSS.0000117134.83018.F7.

Váczi, M., Rácz, L., Hortobágyi, T., Tihanyi, J. (2013). Dynamic contractility and efficiency impairments in stretch-shortening cycle are stretch-load-dependent after training-induced muscle damage. The Journal of Strength \& Conditioning Research, 27 (8), 2171-2179. DOI: 10.1519/JSC.0b013e31827da32c.

Vigotsky, A.D., Lehman, G.J., Beardsley, C., Contreras, B., Chung, B., Feser, E.H. (2016). The modified Thomas test is not a valid measure of hip extension unless pelvic tilt is controlled. PeerJ, 4, e2325. DOI: 10.7717/peerj. 2325.

Vuckovic, G., Dezman, B., Erculj, F., Kovacic, S., Pers, J. (2011). Differences between the winning and the losing players in a squash game in terms of distance covered. In: A. Lees, J.F. Kahn, I.W. Maynard (eds), Science and Racket Sports III, Edition 2. USA: Routledge.

Wakefield, C.B., Halls, A., Difilippo, N., Cottrell, G.T. (2015). Reliability of goniometric and trigonometric techniques for measuring hip-extension range of motion using the modified Thomas test. Journal of Athletic Training, 50 (5), 460-466. DOI: 10.4085/1062-6050-50.2.05.

Whiting, W.C., Zernicke, R.F. (2008). Biomechanics of Musculoskeletal Injury, $2^{\text {nd }}$ ed. USA: Human Kinetics.

Young, S.W., Dakic, J., Stroia, K., Nguyen, M.L., Harris, A.H., Safran, M.R. (2014). Hip range of motion and association with injury in female professional tennis players. The American Journal of Sports Medicine, 42 (11), 2654-2658. DOI: 10.1177/0363546514548852.

Cite this article aS: Trybulec, B., Liput, G., Barłowska-Trybulec. M. (2020). Occurrence and Degree of lliopsoas Muscle Contracture in Regular Male Squash Players. Central European Journal of Sport Sciences and Medicine, 4 (32), 61-68. DOI: 10.18276/ cej.2020.4-06. 